\title{
The Analysis of Film Thickness of Dynamic and Hydrostatic Thrust Bearing with Different Oil-supply Method
}

\author{
Zhao Jianhua ${ }^{a}$, Gao Dianrong ${ }^{b}$ and Cao Daxing ${ }^{c}$ \\ Heibei Provincial Key Laboratory of Heavy Machinery Fluid Power Transmission and Control, \\ Yanshan University, Qinhuangdao 066004, P.R.China

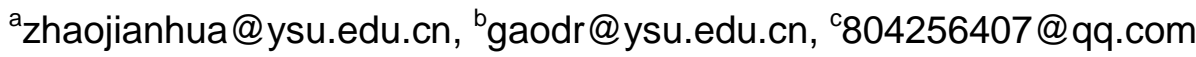

Keywords: Dynamic and hydrostatic thrust bearing; Film thickness; Oil-supply method; Operation stability; Positional accuracy.

\begin{abstract}
Film thickness is the important parameter of dynamic and hydrostatic thrust bearing. The oil film thickness of thrust bearing under the different oil-supply method is different, and it can influence the operation stability and positional accuracy of dynamic and hydrostatic thrust bearing. So this article analysis of the thrust bearing of the thin film thickness in view of the constant pressure, quantitative, PM controller and adaptive supply system of dynamic and hydrostatic pressure thrust bearing, analysis of the influence of structure and process parameters to the thrust bearing, and it provide the theoretical reference for the design and performance analysis.
\end{abstract}

\section{Introduction}

Film thickness is the most important performance parameter of dynamic and hydrostatic thrust bearing. It has the important effects on bearing capacity, static stiffness and operation stability [1-2].

At present, the system of dynamic and hydrostatic pressure thrust bearing can be divided into constant pressure oil supply, quantitative oil supply, PM controller oil supply, constant thickness film servo control oil supply method and so on ${ }^{[3]}$ according to the oil supply method. Constant pressure and quantitative oil supply mainly adopts capillary restrictor, slide valve throttling device for oil supply. When the load increases, the oil flow of the throttle decreases or remain, the change of the film thickness is larger, the stiffness of oil slick is poor [3-4]. The control film of PM controller was adjusted according to pressure. When the load increases, the oil flow of the throttle increases, the stiffness of oil slick increase [5]. Adaptive oil supply system adopts servo control system for precise control of the thrust bearing oil flow. When the load increases, the sensors can pass pressure signal to computer, and after a certain rules increase oil flow, and makes the thin film thickness restoration to the original state, to control of the oil film thickness [6].

Many experts and scholars are devoted to the research of oil-supply method of static pressure system and achieve fruitful research results.

WANG [7] discussed the impact of the initial oil film on static and dynamic performances of liquid thrust bearing oil-supply method and they have been verified by experiment. LIU [8], WANG [9] expounds the advantage of hydrostatic guideway quantitative oil-supply and analyzed the static and dynamic property of long pump oil hydrostatic guideway and it is the development direction of Heavy duty machine tool motion guideway. GUO, LIU [10-11] analyzes the hydrostatic guide ways on heavy duty vertical lathe and $\mathrm{CNC}$ cylindrical grinder, and introduces the quantitative hydrostatic guideway design process and the selection of related parameters.

From the current literature there are no reports of thin film thickness of Static pressure thrust bearing .So this article compared oil film thickness of dynamic and hydrostatic thrust bearing under different oil supply, and analyzes the influence rule of various design parameters on the film thickness of bearing. 


\section{The Way of Constant Pressure Film Thickness}

With flow regulating valve oil supply method as an example analysis of the relationship between thrust bearing oil film thickness and the external load. Transfer function equation of flow regulating valve oil supply thrust bearing

$$
G(s)=\frac{1}{m s^{2}+\Theta_{1} s+\Theta_{0}}
$$

Where $m$-mass of bearing;

$$
\begin{gathered}
\Theta_{1}=\frac{A_{b} A_{e}}{\frac{\bar{B} h_{0}^{3}}{\mu}+\frac{\sqrt{2} A}{4 \sqrt{\rho\left(p_{\mathrm{s}}-p_{0}\right)}}} ; \\
\Theta_{0}=\frac{\frac{3 \bar{B} h_{0}^{2}}{\mu} p_{0} A_{e}}{\frac{\bar{B} h_{0}^{3}}{\mu}+\frac{\sqrt{2} A}{4 \sqrt{\rho\left(p_{s}-p_{0}\right)}}} .
\end{gathered}
$$

The relationship between film thickness and load can be expressed as follow:

$$
\Delta h(\infty)=\frac{\mu}{6 \bar{B} C_{d} m g R_{g} h_{0}^{2}} \Delta f
$$

Among them, $R_{g}$ is flowing resistance of flow regulating valve. Film thickness changes mainly is proportional to the oil viscosity $\mu$, and is inversely proportional to flow load coefficient $\bar{B}$, flow coefficient $C_{d}$, mass $m$, flowing resistance of flow regulating valve $R_{g}$, the initial film thickness $h_{0}$.

Increase flow load coefficient, flow coefficient, mass, flowing resistance of flow regulating valve, initial film thickness, reduce oil viscosity, the film thickness change smaller, increase static stiffness, performance become well. The static stiffness is reduced, the performance is poorer.

\section{Film Thickness of Quantitative Oil-supply Method}

With the oil supply of the control valve as an example analysis of the relationship between thrust bearing oil film thickness and the load. Transfer function equation of the control valve-thrust bearing can be simplified as the second order oscillation link. The expression and transfer function is similar, as shown as Eq. (1).

The relationship between static film thickness and load

$$
\Delta h(\infty)=\lim _{t \rightarrow \infty} \Delta h(t)=\frac{h_{0}}{3 m g} \Delta f
$$

Film thickness is proportional to the initial film thickness $h_{0}$, is inversely proportional to the mass $m$.

Increase mass, reduce initial film thickness, the film thickness change smaller, and increase the static stiffness, performance become well. On the other hand, the film thickness changes big, the static stiffness is reduced, the system performance is poor.

\section{PM Controller Oil-supply Method of Film Thickness}

Transfer function of PM controller-thrust bearing can be expressed as follow: 


$$
G(s)=\frac{1}{m s^{2}+\Theta_{1} s+\Theta_{0}}
$$

Where $m$-mass of bearing;

$$
\begin{gathered}
\Theta_{1}=\frac{A_{b} A_{e}}{\frac{\bar{B} h_{0}^{3}}{\mu}+\frac{\sqrt{2} A}{4 \sqrt{\rho\left(p_{s}-p_{0}\right)}} ;} \\
\Theta_{0}=\frac{3 p_{0} A_{e}}{h_{0}-\frac{\mu q_{0,0}}{\bar{B} p_{s} h_{0}^{2}}\left(K_{r}-1\right)} .
\end{gathered}
$$

The relationship between static film thickness and load

$$
\Delta h(\infty)=\frac{1}{1+\frac{m g}{p_{s} A_{e}}\left(K_{r}-1\right)} \Delta f
$$

Film thickness is proportional to the pump pressure $p_{s}$ and bearing area of oil chambers $A_{e}$ and it is inversely proportional to the mass $m$, PM controller coefficient $K_{r}$.

Increasing quality, coefficient $K_{r}$, reduce system pump pressure, bearing area of oil chambers, the film thickness change smaller, increase the static stiffness, performance become well; On the other hand, the film thickness changes big, the static stiffness is reduced, performance is poorer.

\section{Film Thickness Analysis of Adaptive Oil-supply Method}

Adaptive Oil-supply System changed according to load, and makes the thickness of film tends to be constant. It can be controlled by servo motor + quantitative pump, pump + proportional valve.

Control System of Quantitative Pump + Proportional Valve. The control system of quantitative pump + proportional valve can be made of oil tank, hydraulic pump, proportional valve, pressure gauge, pressure sensor, thrust bearing, A/D converter, D/A converters, comparator, controllers, overflow valve, oil filter, etc.

The operation of the open loop control system is shown as Fig.1. It can be used the external load and the bearing oil cavity area to solve the pressure of the oil cavity; and used the oil cavity pressure and fluid resistance to solve the ideal fluid flow to solve out the required current of the proportional valve, and to keep the film in a state of constant thickness.

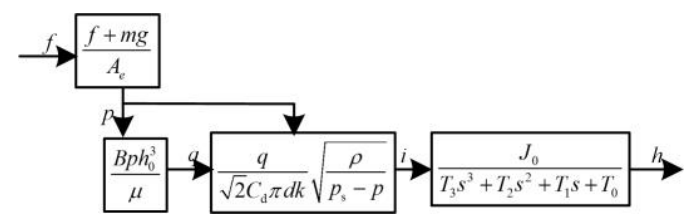

Fig. 1 Operation method of fixed displacement pump + proportional valve + hydrostatic slide open - loop control system

The transfer function of thrust bearing is:

$$
G(s)=\frac{J_{0}}{T_{2} s^{2}+T_{1} s+T_{0}}
$$

Where $J_{0}=C_{d} \pi d k \sqrt{\frac{2}{\rho}\left(p_{s}-p_{0}\right)}$;

$$
T_{0}=\frac{3 \bar{B} p_{0} h_{0}^{2}}{\mu}
$$




$$
\begin{aligned}
& T_{2}=\left(\frac{\bar{B} h_{0}^{3}}{\mu}+\frac{\sqrt{2} C_{d} \pi d k i_{0}}{4 \sqrt{\rho\left(p_{s}-p_{0}\right)}}\right) \frac{m}{A_{e}} ; \\
& T_{1}=A_{b} .
\end{aligned}
$$

Control System of Quantitative Pump + Proportional Valve + Fixed Orifice Bearing. The control system of quantitative pump + proportional valve + fixed orifice bearing is made of fuel tanks, hydraulic pump, proportional valve, fixed throttle mouth, pressure gauge, pressure sensor, thrust bearing, A/D converter, D/A converters, comparator, controllers, overflow valve, oil filter, etc.

The closed loop control system algorithm is shown in Fig.2. It can be used the external load and oil cavity loaded area to solve the pressure of the oil cavity,and can be used the oil cavity pressure and ideal fluid flow of the resistanced oil to solve the idea fluid flow, and can be accorded by the oil flow out with fixed throttle mouth outlet pressure to solve the fixed throttle mouth inlet pressure. Moreover, it can be made the inlet pressure of fixed throttle mouth as the set value and can be used the transfer function of proportional valve current - fixed throttle mouth inlet pressure by the controller, so as to make the control of constant film thickness come true.

The transfer function of thrust bearing system is:

$$
\frac{\Delta H}{\Delta I}=\frac{\Phi_{5}}{\Gamma_{2} s^{2}+\Gamma_{1} s+\Gamma_{0}}
$$

The relationship between the static film thickness and external load is:

$$
\Delta h(\infty)=0
$$

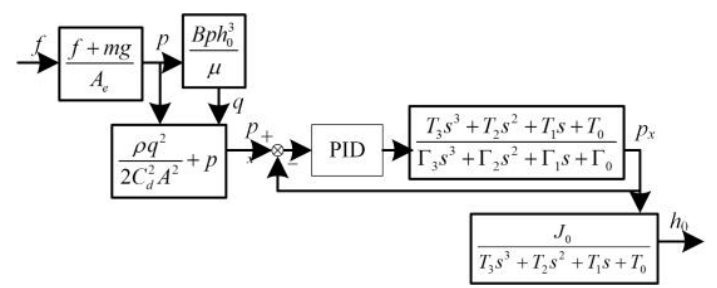

Fig. 2 Operation Rule of Fixed Displacement Pump+ Proportional Valve + Constant Orifices +

\section{Simulation Analysis of Film Thickness of Thrust Bearing with Different Oil-supply Method}

Using the Simulink toolbox which is in Matlab software to build the system block diagram which is under all kinds of oil supply mode of thrust bearing.

Constant pressure oil supply, quantitative oil supply, PM controller oil $\left(K_{l}=3,4,5\right)$, adaptive injection (quantitative pump + proportional valve + fixed throttle control scheme, PID parameters for the $K_{p}=1, K_{i}=40, K_{d}=0$ ) four ways of pulse load (amplitude is $F=30 \mathrm{kN}$, period $T=3 \mathrm{~s}$, width of amplitude is half of the cycle) under the action of thrust bearing of film thickness, as shown in Fig.3.

Under continuous pulse load, flow under the way of constant pressure oil supply system can be changed with the load increase and decrease, film stiffness also chang the worst, so the film thickness changes the largest , $3.766 \mu \mathrm{m}$. 


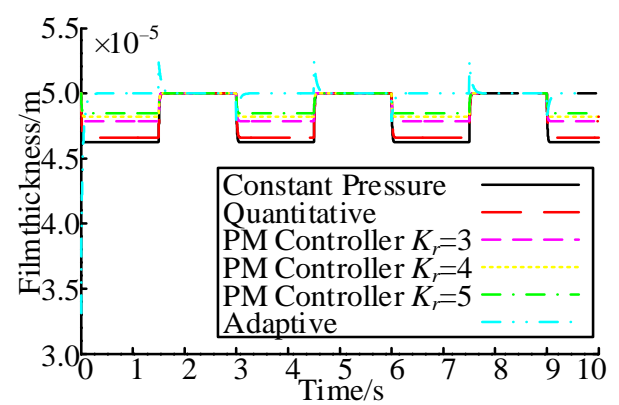

Fig. 3 Oil film thickness of hydrostatic slide with different supplying methods

With the external load increased,the traffic flow is kept to be constant by the way of quantitative oil flow.at the sanme time film stiffness is increased, so the film thickness change is lessl, so as to $3.401 \mu \mathrm{m}$

the oil flow rate which is under PM controller is increased, with the increased as the external load, and the less the thickness of the thin film becomes, the greater changes the thin film stiffness have greatly improved.

Moreover, with the change of the $K_{r}$ increased, film thickness is decreased ( When $K_{r}=3 ; \Delta h=$ $2.1261 \mu \mathrm{m}$; when $K_{r}=4, \Delta h=1.79 \mu \mathrm{m}$; when $\left.K_{r}=5, \Delta h=1.546 \mu \mathrm{m}\right)$. The fuel system supply which is under the adaptive oil supply mode can be adjusted themselves, which is under the flow with the load change. So as to make the film can maintain constant thickness or approximate constant thickness.

The oil film thickness can be kept as the standard of engineering allowed range with the control of PM controller oil and the daptive fuel supply scheme.

Under the PM controller oil he design parameter $K_{r}$ tends to infinity, the film thickness changes as:

$$
\lim _{K_{r} \rightarrow \infty} \Delta h(\infty)=\lim _{K_{r} \rightarrow \infty} \frac{\Delta f}{1+\frac{m g}{p_{s} A_{e}}\left(K_{r}-1\right)}=0
$$

When parameter $K_{r}$ is increasesd, the film thickness change is decreased; When $K_{r}$ is tended to be infinity, film thickness change becomes zero, so as to be kept the constant state of film thickness, moreover to achieve the same effect, just as the adaptive fuel supply scheme.

\section{Conclusion}

The film thickness which is under the constant pressure supply thrust bearing oil film thickness change is the largest. The film thickness which is under the amount of fuel supply schemes change is less than the former. The film thickness which is under the control of PM controller oil changes is less than the formers. The film thickness which is under the adaptive fuel supply scheme, ideally it can realize constant thickness of thin film.

\section{Acknowledgements}

The project was financially supported by Young Teachers Independent Study Project of Yanshan University (Grant No. 13LGB003) and Research and Development of Science and Technology Plan of Qinhuangdao City (201401A002).

\section{References}

[1] O. Dalane, K. F. Faye, S. Lset . Nonlinear Coupled Hydrostatics of Arctic Conical Platforms [J]. Journal of Offshore Mechanics and Arctic Engineering, 2011, 134(2): 73-82.

[2] K. Khorshidi. Effect of Hydrostatic Pressure on Vibrating Rectangular Plates Coupled with Fluid [J]. Scientia Iranica, 2010, 17(6): 415-429.

[3] V. M. Phalle , S. C. Sharma , S. C. Jain, et al. Performance Analysis of a 2-lobe Worn 
Multirecess Hybrid Journal Bearing System using Different Flow Control Devices [J]. Tribology International, 2012, (52): 101-116.

[4] S. C. Sharma , V. M. Phalle , S. C. Jain . Performance of a Noncircular 2-lobe Multirecess Hydrostatic Journal Bearing with Wear [J]. Industrial Lubrication and Tribology, 2012, 64(3): 171-181.

[5] A. V. Beznsov, Y. N. Drozdov, M. A. Antonenkov, et al. Tribology of Hydrostatic Bearings of Coolant Pumps of Fast Neutron Reactors with Lead Coolant [J]. Journal of Friction and Wear, 2012, 33(5): 338-344.

[6] F. Tomoya, M. Atsushi, Y. Kazuo. Expermental Characterization of Disturbance Force in a Linear Drive Sustem with High-precision Rolling Guideways [J]. International Journal of Machine Tool and Manufacture, 2011, (51): 104-111.

[7] Y. Wang, Z. C. Pang. Effect of Design Gap of Quantitative Oil-supply Hydrostatic Bearing on Static and Dynamic Characteristics [J]. Machinery, 1990, 17(6): 17-19. (In Chinese)

[8] C. X. Liu. Design of Constant Flow Control on Liquid Hydrostatic Guide-way [J]. Machine Tool \& Hydraulics, 2008, 36(7): 125-128. (In Chinese)

[9] H. Wang, X. D. Chen. Design and Analysis of Constant Flow Control on Hydrostatic Guide-way [J]. Equipment Manufactring Technology, 2011, (7): 66-68. (In Chinese)

[10] Y. Y. Guo. Design of Hydrostatic Slide of Vertical Lathe [J]. Mechanical Engineer,, 2011, (6): 118-119. (In Chinese)

[11] J. S. Liu. Design of Closed-type Hydrostatic Slide of Wheel Head of NC Cylindrical Grinder [J]. Precise Manufacturing \& Automation, 2011, (4): 36-39. (In Chinese) 